\title{
Gamma-ray bursts
}

\author{
Frédéric Daigne* \\ Institut d'Astrophysique de Paris \\ E-mail: daigne@iap.fr
}

Gamma-ray bursts are the most powerful explosions in the Universe. They appear as brief bursts of gamma-rays followed by an afterglow at longer wavelengths. They involve a complex physics with a relativistic ejection by a new-born compact stellar mass source, and several phases of emission associated to internal dissipation in the ejecta and to the interaction of the ejecta with its environment. I will review the observations of these phenomena and discuss our current understanding of their physical origin.

Frontiers of Fundamental Physics 14 - FFP14,

15-18 July 2014

Aix Marseille University (AMU) Saint-Charles Campus, Marseille

${ }^{*}$ Speaker. 